Ann. Sci. forest., 1968, 25 (3), 189-192.

NOTE TECHNIQUE

\title{
PREMIERS ESSAIS D'ÉLEVAGE DES LARVES D'HYLOBIUS ABIETIS SUR MILIEU SYNTHÉTIQUE
}

\author{
C.-B. MALPHETTES \\ avec la collaboration technique de A. Serpillon et J. Muller \\ Laboratoire d'Entomologie, \\ Centre national de Recherches forestières, 54-Nancy \\ Institut national de la Recherche agronomique
}

SOMMAIRE

L'auteur développe la technique qui lui a permis d'élever des larves de Hylobius abietis L. (Coleoptera, Curculionidae) sur un milieu reconstitué à partir d'écorce de Pin sylvestre et de substances chimiques définies.

\section{INTRODUCTION}

Dans le cadre de nos recherches entreprises pour l'étude du développement de Hylobius abietis L., nous avons été amenés à élever des larves pour déterminer le nombre de stades larvaires et leur durée. Jusqu'à présent nous avons utilisé une technique dérivée de celle mise au point par WARREN pour un hylobe nord-américain (Hylobius warreni), et qui nous a donné satisfaction. Cependant cette méthode a le gros inconvénient de demander énormément de manipulations, la durée du développement exigeant environ 3 mois à $20^{\circ} \mathrm{C}$ de l'œuf à la formation de l'adulte. Ces manipulations ont entraîné une grande hétérogénéité dans la durée du développement et aussi une mortalité quelquefois importante. Mais finalement le plus gros reproche que l'on puisse faire à cette technique est qu'elle demande trop de temps ce qui empêche l'élevage simultané de nombreuses larves.

Depuis plusieurs années l'élevage en milieu artificiel synthétique ou semi-synthétique se développe dans de nombreuses recherches portant surtout sur des Lépidoptères ou des Diptères. GuENNELON (1967) dans une synthèse des travaux en cours cite les principaux insectes sur lesquels des 
essais ont été tentés. Cet auteur précise les principaux avantages que l'on peut attendre de l'alimentation artificielle des insectes ainsi que les différentes formes sous lesquelles les milieux sont présentés aux insectes. HousE (1967) pour sa part, résume les caractéristiques contenues dans près de 200 publications. Ce sont surtout les Américains et les Canadiens qui, au cours des dernières années, se sont attaqués au problème de l'élevage des xylophages (au sens large du terme : insectes vivants aux dépens de l'écorce ou du bois), sur des milieux reconstitués.

C'est en nous inspirant de toutes ces méthodes que nous avons essayé de mettre au point un milieu semi-artificiel pour l'élevage des larves de l'hylobe. Cette technique dans notre esprit, devrait permettre une plus grande homogénéité des élevages et une demande de temps moins élevée que dans le cas de la méthode que nous avons utilisée jusqu’à présent.

\section{MÉTHODES}

Les travaux de Schmidt (1966) sur Dendroctonus pseudotsugae Hopk. et ceux de Clark (1965) sur Dendroctonus frontalis Zıмm. ont été le point de départ de nos essais.

\section{1. - Technique de préparation du milieu}

Au début nous avions essayé de composer un milieu holidique (1) au sens de DougherTy (1959) en partant de cellulose pure, substance chimique facilement disponible puisque servant en chromatographie. Cependant cet essai a été un échec, aucune larve n'ayant réussi ni à s'installer, ni a fortiori à se développer. Par ce moyen nous espérions pouvoir être indépendants du milieu végétal et surtout des exploitations de résineux et en particulier de Pin svolvestre. Devant cet échec, nous avons dû nous retourner vers du matériel se rapprochant plus du " matériel naturel » et constituer, comme le dit GuenNelon (1967), un intermédiaire entre un milieu oligidique et un milieu méridique.

\section{TABLeAU 1}

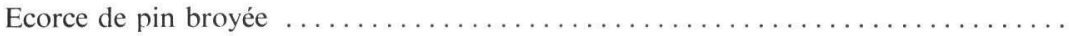

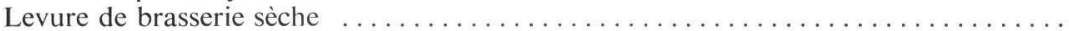

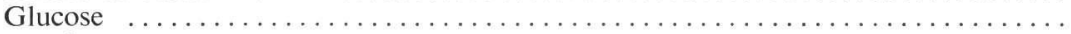

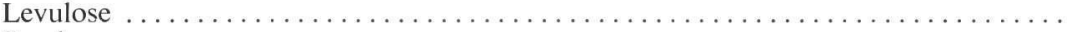

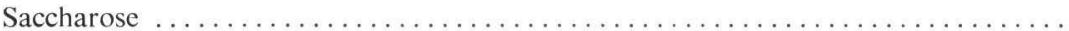

$3,00 \mathrm{~g}$

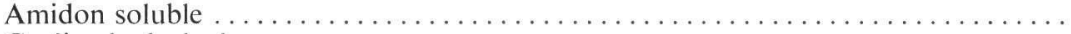

$2,00 \mathrm{~g}$

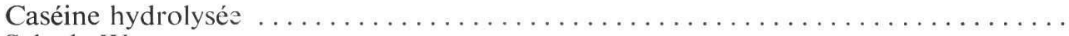

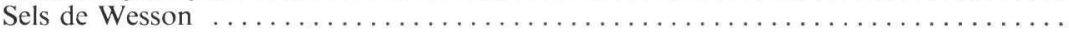

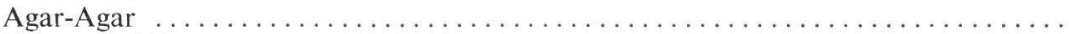

$1,00 \mathrm{~g}$

$6,00 \mathrm{~g}$

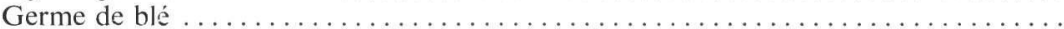

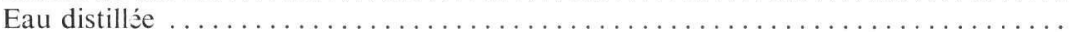

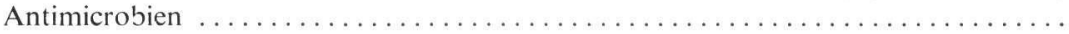

La composition de ce milieu est celle donnée sur le tableau I. Les larves dans la nature se développent dans l'écorce des racines des souches de Pin ou d'Epicéa, fraîchement abattus. Il était difficile de s'approvisionner avec cette écorce. Depuis le début de nos études nous l'avons toujours remplacée par de l'écorce de branches et le développement a paru normal. Pour la préparation de l'écorce destinée à la confection du milieu, sur les chantiers d'abattage, nous prélevons des rondins frais de cimes, choisis de façon à ce que l'écorce comporte le moins possible de zone liégeuse. Ces rondins font en moyenne 20 à $30 \mathrm{~cm}$ de diamètre et proviennent de Pins sylvestres de 100 à 150 ans; ils

(1) Un milieu qui ne renferme que des matériaux bruts alimentaires est dit oligidique, un milieu holidique par contre, ne contient que des substances chimiques bien définies; enfin le milieu qui se compose à la fois des matériaux bruts et des substances chimiques est qualifié de méridique. 
ont une «écorce » utilisable de 2 à $3 \mathrm{~mm}$ d'épaisseur. Le terme d'écorce est d'ailleurs pris au sens forestier puisqu'il s'agit en fait aussi bien du liber que de l'écorce proprement dite. Au laboratoire, l'écorce de ces rondins est prélevée, découpée en languettes de quelques centimètres après avoir été débarrassée de toutes les zones liégeuses. Ces languettes sont ensuite placées dans le bol d'un moulin à café électrique et broyées pendant $2 \mathrm{mn}$ environ à grande vitesse. On obtient ainsi de petites particules de $1 / 2 \mathrm{~mm}$ de large sur 2 à $3 \mathrm{~mm}$ de longueur. Ce broyat est ensuite stocké dans des récipients hermétiquement clos au réfrigérateur jusqu'au moment de l'utilisation. Ce broyage s'effectue correctement si on prend soin de découper l'écorce en languettes. L'écorce ainsi prélevée contient en moyenne $65 \%$ d'eau et a un $\mathrm{pH}$ voisin de 5.

Pour préparer le milieu proprement dit, on porte à ébullition la quantité d'eau nécessaire après y avoir ajouté les différents sucres et l'agar-agar. L'ébullition est maintenue pendant $5 \mathrm{mn}$. Ensuite on laisse refroidir au bain-marie jusqu'à $50^{\circ} \mathrm{C}$ avant d'ajouter les sels de Wesson, la levure de brasserie, la caséine, le germe de blé et l'anti-microbien. Ce dernier est apporté sous la forme d'une solution de $5 \mathrm{~g}$ de Para-Hydroxybenzoate de Méthyle dans de l'alcool éthylique à $95{ }^{\circ} \mathrm{GL}$ nour faire $100 \mathrm{ml}$. Un mélange homogène est obtenu après brassage à l'aide d'un mixer plongeant. L'écorce de pin broyée est ensuite ajoutée et le mélange obtenu par agitation avec une spatule. Quand le milieu est terminé, il est disposé à la spatule dans les récipients en plastique et on laisse solidifier pendant $1 \mathrm{~h}$ à l'air libre. Les récipients sont ensuite fermés et stockés au réfrigérateur jusqu'à leur utilisation.

\section{2. - Mise en place de l'élevage}

Ce sont les jeunes larves nouveau-nées qui sont introduites dans le milieu. Les adultes pondent dans des morceaux de branches de Pin. Ces branches sont écorcées pour recueillir les oeufs. Ces derniers sont ensuite disposés sur des feuilles de cellulose humide à raison d'un par boîte ; ceci évite aux jeunes larves de se mordre mutuellement quand elles se rencontrent. Les éclosions qui ont lieu au bout d'une semaine à $20^{\circ} \mathrm{C}$ sont ensuite suivies jour par jour et les jeunes larves qui viennent d'éclore sont introduites dans le milieu.

Ces larves étant apodes, il faut les introduire directement au sein du milieu pour qu'elles puissent creuser leurs galeries. Dans ce but, un trou conique de $1 \mathrm{~mm}$ de diamètre environ est fait dans le milieu solidifié. La larve manipulée au pinceau, est introduite et le trou est rebouché derrière elle. Dans une boîte de 4,5 $\mathrm{mm}$ de diamètre, on n'introduit que 2 larves. En effet sans parler à proprement dit de cannibalisme, il semble que les larves qui se rencontrent se mutilent, ce qui entraine une forte mortalité. Deux larves pour un récipient de la taille ci-dessus est un maximum quant au rendement. Les larves s'installent bien dans le milieu et forent une galerie. Le plus souvent une des parois de cette galerie est constituée par les parois du récipient. Il arrive souvent aussi que les larves en creusant la galerie, gagnent la surface du milieu. Dans ce cas, le plus fréquemment la larve creuse une galerie non couverte à la surface mais finalement le sillon devient trop impertant et la larve n'arrive plus à creuser le milieu, faute de point d'appui : elle tourne en rond à la surface et cela entraîne un retard ou un arrêt dans le développement.

Compte tenu de ces remarques nous avons modifié le protocole d'élevage. Nous utilisons maintenant des récipients beaucoup plus plats $(6 \mathrm{~mm}$ de hauteur totale) et rectangulaires ; cette épaisseur permet à la larve, à partir de la moitié de son développement, de prendre directement appui sur le couvercle pour s'alimenter. De plus les larves sont changées dès qu'elles semblent avoir consommé au maximum le milieu mis à leur disposition, la dimension du récipient augmentant à chaque changement. En outre, au bout de 15 jours à 3 semaines de développement, les larves sont élevées individuellement.

Dans ces conditions, nous avons obtenu des adultes bien conformés, par comparaison avec ceux capturés dans la nature, en deux mois et demi. Ces adultes ont même commencé à pondre.

\section{CONCLUSIONS}

Cette courte note n'a pour but que de signaler le point de départ de nos recherches actuellement entreprises pour élever Hylobius abietis sur milieu reconstitué. Il reste bien présent à notre esprit que tout est encore très perfectible. Mais à notre connaissance, c'est la première fois qu'un tel essai est tenté sur cet insecte.

Actuellement nos efforts portent sur deux points techniques : le récipient et les colonies bactériennes. Dans le choix du récipient, il faut tenir compte de deux faits : les larves demandent des 
points d'appui pour consommer l'aliment et ensuite le développement de la larve est long. On peut fournir un point d'appui aux larves en les élevant dans des boîtes peu épaisses où le fond et le couvercle peuvent permettre à l'insecte de creuser une galerie. Le développement de longue durée demande lui, que l'on change plusieurs fois la larve de milieu sinon des fermentations s'installent.

D'autre part, nous nous efforçons d'éliminer les proliférations bactériennes qui se trouvent très souvent à la surface du milieu, qu'elles occupent très rapidement. Quand les larves se déplacent sur ces colonies, elles meurent. Il n'est pas encore établi si la mort est due à une quelque action pathogène des bactéries ou si elle est due à une asphyxie provoquée par le caractère huileux de la colonie, qui peut alors obturer les stigmates.

Il apparaît donc que l'élevage de Hylobius abietis sur milieu reconstitué est possible. Il faut maintenant améliorer considérablement la technique. Ensuite peut-être sera-t-il possible d'utiliser ce procédé pour l'élevage d'autres Coléoptères forestiers ; nous pensons essentiellement au genre Pissodes mais aussi aux Scolytidae, comme cela a déjà été fait en Amérique du Nord. Si un telélevage devenait «rentable », cela pourrait intéresser tous ceux qui actuellement, s'occupent plus particulièrement de lutte biologique. La multiplication des parasites ou des maladies des insectes de ce groupe pourrait être alors facilitée.

Reç pour publication en juin 1968

\section{SUMMARY}

First EXPERIMENTAL REARINGS of Hylobius abietis laRVAe on A SYNTHETICAL MEDIUM

The rearing of the insects in the bark living raises numerous technical difficulties, some of them are tentatively solved. The larvae of the Large Pine Weevil Hylobius abietis L. (Coleoptera, Curculionidae) were reared on a synthetical medium.

The writer explains in details the composition and the making of this medium. He points out the difficulties he has encountered : bacterial development, requirement of a purchase for the larva, long duration of the growth.

\section{ZUSAMMENFASSUNG}

ERSTE AUfZUCHTVERSUCHE MIT DEN LARVEN VON Hylobius abietis IN SYNTHETISCHEM MEDIUM

Die Züchtung der Larven, der in der Rinde lebenden Insekten, verursacht zahlreiche technische Schwierigkeiten. Der Verfasser versuchte einige davon zu beschliessen; dazu zog er Larven des Rüssenlkäfers Hylobius abietis L. (Coleoptera, Curculionidae) in einem synthetischen Nährmittel auf.

Er beschreibt die Zusammensetzung dieses Medium in allen Einzelheiten und auch wie es herstellen kann. Darauf hebt er die Schwierigkeiten auf die er stoss, hervor : Bacterien Ausbildung, Erfordernis eines für die Larva Stïtzpunkt, lange Dauer der Entwicklung der Insektenbrut.

\section{RÉFÉRENCES BIBLIOGRAPHIQUES}

Clark E.-W., 1965. - An artificial diet for the southern pine beetle and other bark beetles. U.S. Forest. Serv. Res. Note SE 45.

Dougherty E.-C., 1959. - Introduction to axenic culture of Invertebrate Metazoa : a goal. Ann. N.Y. Acad. Sci., 77, 27-54.

Guennelon G., 1967. - L'alimentation artificielle chez les insectes. Rev. Zool. agric. appl., 66, (1-3), 20-28.

House H.-L., 1967. - Artificial diets for insects : a compilation of references with abstracts. Inform. Bull., 5, Res. Inst. Canad. Dept. Agric. Belleville, Ontario.

Schmidt F.-H., 1966. - Two artificial (oligidic) media for the Douglas-Fire beetle, Dendroctonus pseudotsugae Hopkins. Canad. Entomol., 98, (10), 1050-1055. 